\title{
Surface Texture Characterization Using Optical and Tactile Combined Sensor
}

\author{
Kenta Takahashi, ${ }^{1}$ Takashi Abe, ${ }^{1}$ Masanori Okuyama, ${ }^{2}$ \\ Haruo Noma, ${ }^{3}$ and Masayuki Sohgawa ${ }^{1 *}$ \\ ${ }^{1}$ Graduate School of Science and Technology, Niigata University, \\ 8050 Ikarashi 2-no-cho, Nishi-ku, Niigata 950-2181, Japan \\ ${ }^{2}$ Institute for NanoScience Design, Osaka University, \\ 1-3 Machikaneyama-cho, Toyonaka, Osaka 560-8531, Japan \\ ${ }^{3}$ College of Information Science and Engineering, Ritsumeikan University, \\ 1-1-1 Nojihigashi, Kusatsu, Shiga 525-8577, Japan
}

(Received Octorber 26, 2017; accepted February 7, 2018)

Keywords: optical proximity sensor, tactile sensor, combination sensing, texture characterization

Measurements of the surface texture of objects, including optical and tactile features, using a multimodal micro-electromechanical systems (MEMS) sensor are reported in this paper. The proposed MEMS sensor has two functions in its structure: light sensitivity of the MOS structure in the $\mathrm{Si}$ substrate and force sensitivity of the strain resistance gauge on microcantilevers embedded in the elastomer. Deflection of the cantilever, induced by an applied force, can be detected as a DC resistance change of the strain gauge film, which depends on the tactile texture including hardness, thickness, and surface roughness of the object. On the other hand, reflected light from the object, detected as $\mathrm{AC}$ impedance change at $5 \mathrm{MHz}$, depends on the color of the object. It is confirmed that the resistance and impedance changes correlate with the physical and optical properties of the object, respectively. Therefore, it has been demonstrated that the surface texture of the object, including optical and tactile features, can be characterized using a single MEMS sensor.

\section{Introduction}

In recent years, texture designs for high-value-added products have become very important. ${ }^{(1)}$ Texture is also an important factor in choosing a custom-made product on the basis of its visual and tactile modalities. When possible, customers seem to want to confirm the texture of the products through visual as well as haptic feedback. It is known that the texture of the object is perceived through multimodal senses including touch and vision. ${ }^{(2)}$ The human brain perceives cross-modal information between touch and vision. ${ }^{(3)}$ Customers can visually verify the texture of a product through an online catalog; however, they cannot evaluate the feel of the product because of the lack of haptic information. Some tactile displays to reproduce tactile texture have been proposed; however, visual information is not included. ${ }^{(4)}$ Hence, cross-modal sensing of tactile and visual information is necessary to evaluate the texture of a product more precisely.

*Corresponding author: e-mail: sohgawa@eng.niigata-u.ac.jp http://dx.doi.org/10.18494/SAM.2018.1786 
Typically, the texture of an object is characterized by human sensory evaluation. ${ }^{(5)}$ However, sensory evaluation requires many test subjects and significant testing time. Furthermore, it lacks quantitativeness and reproducibility because it is a subjective estimation. Recently, many kinds of sensors imitating the five senses have been developed in the field of robotics, and it is the same in the case of tactile sensors. ${ }^{(6)}$ There are various kinds of tactile sensors, such as optical, piezoresistive, piezoelectric, capacitive, and elastoresistive sensors. ${ }^{(7-11)}$ Some approaches to the quantitative characterization of texture using these sensors have been attempted as a replacement for sensory testing. Mazid and Russell measured texture as surface roughness by scanning with an opto-tactile sensor. ${ }^{(12)}$ Tsuchimi et al. measured human skin conditions with a haptic sensor system. ${ }^{(13)}$ Asaga et al. developed a tactile evaluation method based on a human tactile perception mechanism. ${ }^{(14)}$ As another approach for visual texture characterization, Abe et al. measured quantitative glossiness and transparency from pictorial images $^{(15)}$ and Motoyoshi et al. measured surface quality using photographs. ${ }^{(16)}$ However, no sufficient alternative sensing method with sensitivity to both tactile and visual features has yet been realized because of the cross-modality of human senses, as mentioned earlier.

In our previous works, we developed a multiaxial MEMS tactile sensor that can detect normal and shear forces using microcantilevers with a $\mathrm{NiCr}$ strain gauge film. ${ }^{(17-23)}$ This sensor has been applied to tactile texture measurement, thereby enabling qualitative classification of many kinds of objects. ${ }^{(24-27)}$ However, more quantitative tactile characterization is required to evaluate physical properties such as the hardness, thickness, and roughness of an object. Moreover, it has been demonstrated that our developed MEMS sensor has multimodal sensitivity to force and light and can detect the proximity of the sensor to the target from the intensity of reflected light with simultaneous touch force detection. ${ }^{(28-30)}$ One of the benefits of using the single sensor sensitive to light and force is space saving and miniaturization to easily install multiple sensors in a robotic fingertip. Another benefit is that the touch force and color can be measured simultaneously even in the case of changing color upon contact, such as on human skin. We have reported the results of a basic study on the characterization of the surface texture of various objects including tactile and optical features. ${ }^{(31-33)}$ In this study, the characteristics of the tactile sensor output in relation to the object's hardness, thickness, and surface roughness have been examined in more detail. Furthermore, optical texture, including color and surface reflection, has been characterized using the reflected light from the object.

\section{Materials and Methods}

\subsection{Structure of MEMS sensor}

Figure 1 shows a conceptual illustration of the MEMS sensor, a microscopic image of the microcantilevers, and the cross-sectional structure of the microcantilever. ${ }^{(19-23)}$ The microcantilevers are embedded in polydimethylsiloxane (PDMS). The microcantilever has a sloping form in order to be sensitive to both normal and shear forces. Three microcantilevers are located within a $\varnothing 1 \mathrm{~mm}$ circle, as shown in Fig. 1(b). The microcantilevers were fabricated on the Si-on-insulator (SOI) wafer by the surface MEMS process. Thin films of $\mathrm{Si}_{3} \mathrm{~N}_{4}, \mathrm{Cr}$, 


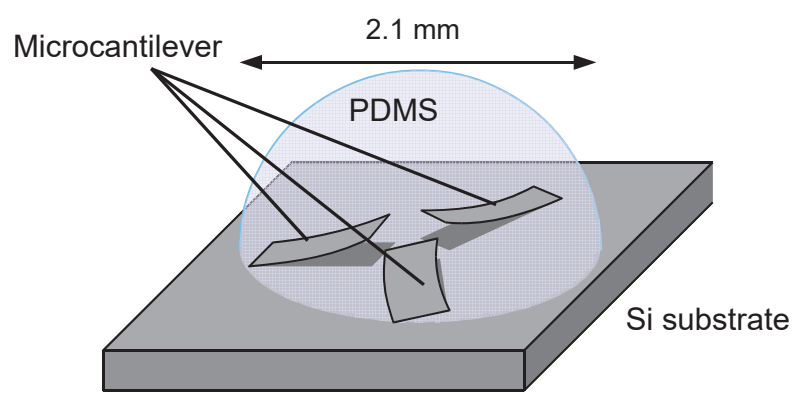

(a)

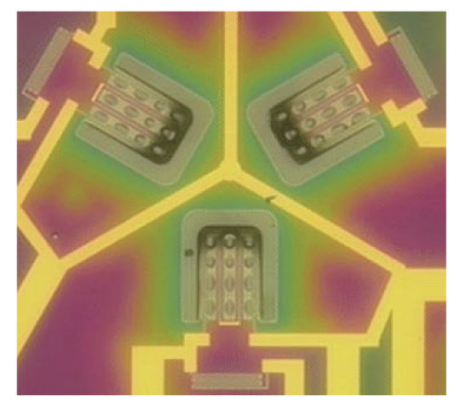

(b)

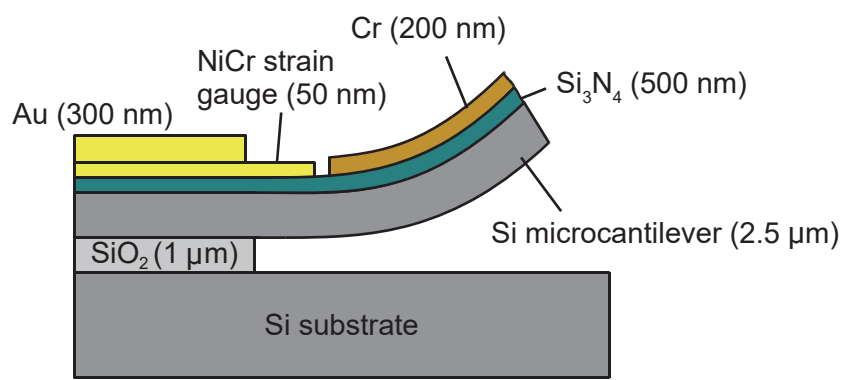

(c)

Fig. 1. (Color online) (a) Conceptual illustration of tactile sensor, (b) microscopic image of the microcantilevers, and (c) cross-sectional structure of a microcantilever (values shown in parentheses are thickness of each layer).

$\mathrm{NiCr}$, and $\mathrm{Au} / \mathrm{Cr}$ were formed, as shown in Fig. 1(c), for insulation, shape control, strain gauge, and electrode, respectively. The microcantilevers were covered with hemispherical PDMS after a PDMS film was spin-coated on them.

\subsection{Principle of optical sensing}

Figure 2 shows a conceptual diagram of light sensing. When the sensor surface is irradiated with light, carrier pairs are generated by photoabsorption in the upper Si layer of the SOI wafer since the transmittance of the PDMS is around $85 \%$ in the visible light range. ${ }^{(34)}$ The AC impedance between electrodes at high frequency includes the Si resistance and the capacitance of the $\mathrm{Si}_{3} \mathrm{~N}_{4}$ layer and the depletion layer in $\mathrm{Si}$. The resistivity and depletion depth in $\mathrm{Si}$ decrease with the increase of carrier density. ${ }^{(29)}$ Thus, the intensity of light can be measured as the impedance of the sensor. Figure 3 shows the relative impedance change (at $5 \mathrm{MHz}$ ) between electrodes as a function of LED light illuminance. The impedance decreases with the increase in the illuminance of LED light. It is found that the fabricated sensor is sensitive to light illuminance; however, it does not have wavelength selectivity because Si broadly absorbs light with wavelengths shorter than $1.1 \mathrm{~mm}$, which is the visible light range. ${ }^{(35)}$ Hence, to detect the object color, light sources or color filters of the three primary colors (red, green, blue) are needed. Although the color is generally represented by the RGB model for sensing and display, we have employed the hue, saturation, value (HSV) model for the color representation because 


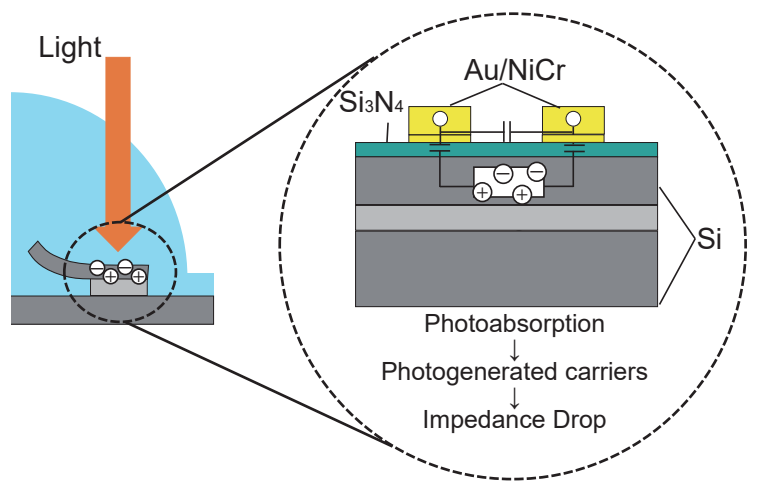

Fig. 2. (Color online) Conceptual diagram of light sensing by photoabsorption in the Si layer.

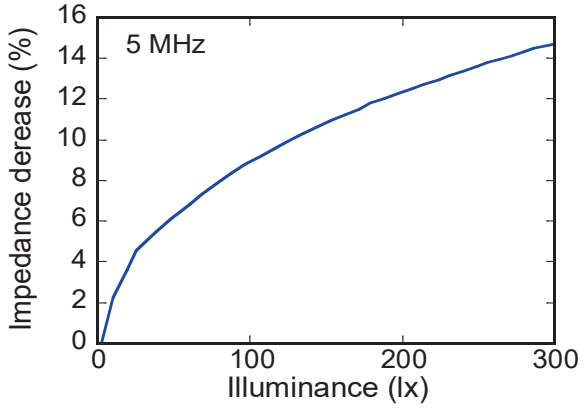

Fig. 3. (Color online) Impedance decrease of the sensor as a function of light illuminance.

it is more intuitive and perceptually relevant than the RGB model. Figure 4 shows the decrease in the relative impedance of the sensor, induced by the reflection of probe light with threecolor light (center wavelengths are 470, 525, and $621 \mathrm{~nm}$ for red, blue, and green, respectively) from LEDs as a function of the hue of the object (color-printed paper). When the color of the probe light is similar to the color of the target, the reduction in the impedance is relatively large because other colors are absorbed by the object, whereas similar colors are reflected. Thus, we can evaluate the colored feature using correlation between the impedance of the sensor and the color of the probe light.

\subsection{Principle of tactile sensing}

Figure 5 shows the conceptual illustration of the multiaxial force sensing system. Deflection of the microcantilever by applied force is detected as a resistance change of the $\mathrm{NiCr}$ thin film strain gauge. When normal force is applied to the surface of the sensor, microcantilevers are deflected upward because of lateral deformation of the PDMS. On the other hand, in the case of shear force application, the microcantilevers are deflected in the shear force direction. Therefore, multiaxial force can be detected because the deflection of the cantilevers depends on the force direction. Figures $6(\mathrm{a})$ and $6(\mathrm{~b})$ show the relative resistance change of the strain gauge on the microcantilever as a function of normal and shear forces, respectively. The resistance changes are obviously proportional to both normal and shear forces. In addition, the slope of resistance change to shear force is dependent on the direction of shear force, as shown in Fig. 6(b). The slope becomes maximum when the shear force and cantilever directions are parallel.

\subsection{Characterization method of surface texture of object}

Figure 7 illustrates the experimental methods of the tactile characterization of the object. The measurement methods involve indentation and pull-up testing [Fig. 7(a)], and slide testing [Fig. 7(b)]. In the indentation and pull-up test, the object is indented onto the sensor surface to a depth of $200 \mathrm{~mm}$ and then pulled up. In the sliding test, the sensor is moved at a speed of 100 


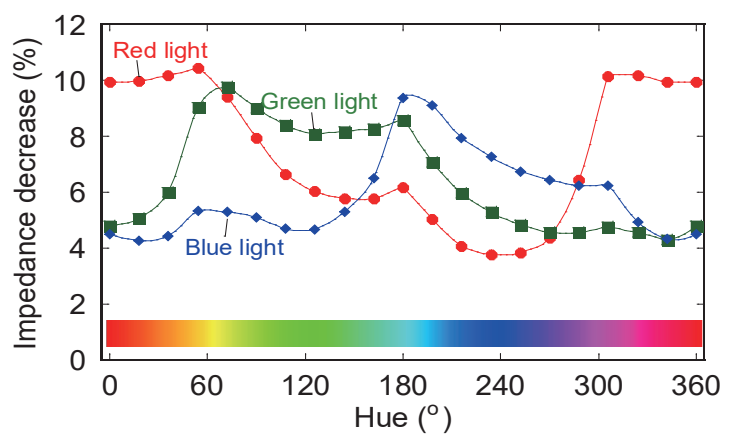

Fig. 4. (Color online) Change in relative impedance of the sensor, induced by reflection, from the object, of the three-color probe light as a function of hue value.

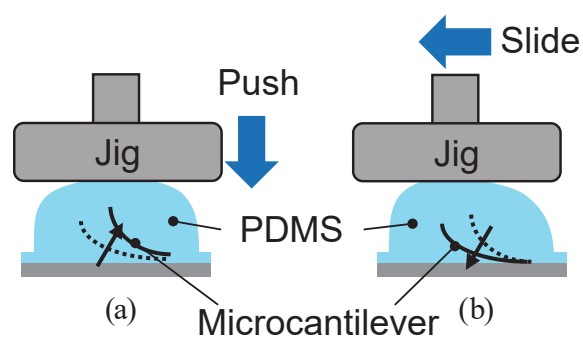

Fig. 5. (Color online) Conceptual diagrams of sensing of multiaxial force: (a) normal force and (b) shear force.

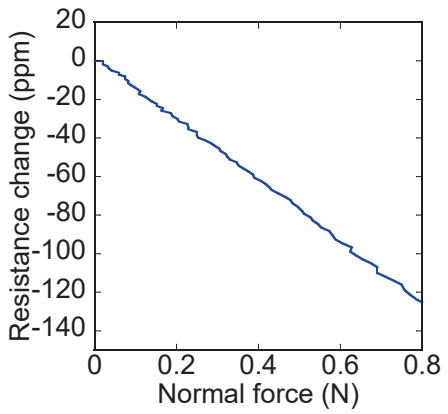

(a)
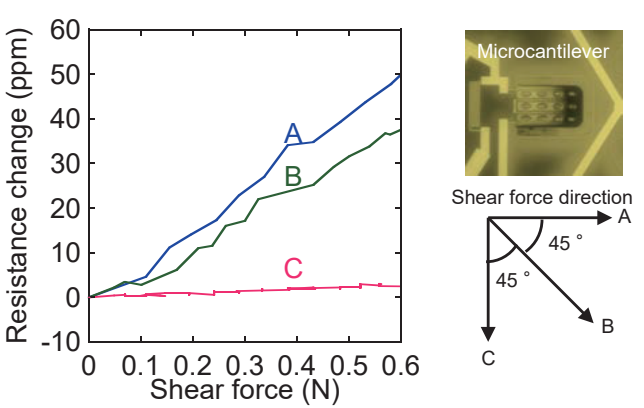

(b)

Fig. 6. (Color online) Change in relative resistance as a function of (a) normal and (b) shear forces.

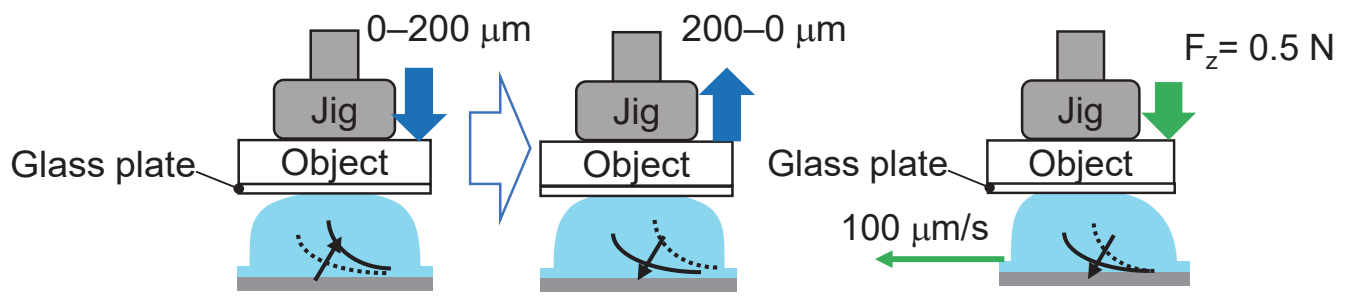

(a) (b)

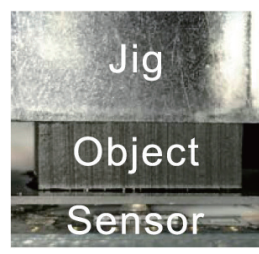

(c)

Fig. 7. (Color online) Schematic illustrations of measurement methods for tactile characterization of the object: (a) indentation and pull-up test, (b) sliding test, and (c) photograph of the measurement setup.

$\mathrm{mm} / \mathrm{s}$ horizontally after indenting the object into the sensor surface with a normal force of 0.5 $\mathrm{N}$. The movement direction in the sliding test is set as the direction with the highest gradient of resistance change, because the resistance change depends on the direction of shear forces, as shown in Fig. 4(b). The change in resistance over time has been characterized in these tests. As objects for texture measurement, various kinds of rubber (chloroprene, silicone, polyurethane, and others) with hardness of 25-90 (Shore A hardness; Hs) and thickness of 1-10 mm were employed, where $H s$ of the rubber eraser and tire rubber are 30-40 and 60-70, respectively. To standardize the surface characteristics of the object, they were covered with a thin flat glass plate $\left(R_{z}\right.$ 
$<30 \mathrm{~nm}$ ). In addition, glass plates with surface roughness of $R_{z}=0.48-3.49 \mathrm{~mm}$ were prepared by hydrogen fluoride etching to investigate the effect of the object surface roughness.

Figure 8 shows a schematic illustration of the measurement system for the optical characterization of the object. The object covered with the flat glass plate is irradiated with three-color probe light from an LED installed at a position lateral to the multimodal sensor. The sensor is $10 \mathrm{~mm}$ away from the object and the LED because the reflected light easily irradiates the sensor. The impedance of the sensor depends on the surface optical characteristics, including reflectivity and color of the object, as shown in Figs. 3 and 4. In this study, the rate of change from the impedance (at $5 \mathrm{MHz}$ ) without light irradiation (in a dark environment) was measured.

\section{Results and Discussion}

\subsection{Characterization of tactile features}

Figures 9 and 10 show the changes in the relative resistance of the sensor, $\Delta R_{p} / R$, as a function of indentation depth, and $\Delta R_{S} / R$ as a function of time when the sensor is slid on the objects, respectively. It is found that the resistance changes in Figs. 9 and 10 depend on the characteristics of the object, including hardness, thickness, and surface roughness. In the case of indentation and pull-up testing, $\Delta R_{p} / R$ decreases with the increase of indentation depth. It is found that the maximum $\Delta R_{p} / R$ at the indentation depth of $200 \mathrm{~mm}$ increases and decreases monotonically with hardness and thickness, respectively. On the other hand, its dependence on surface roughness is complicated; it increases from $R_{z}=0.03$ to $0.48 \mathrm{~mm}$ and decreases from $R_{z}=0.48$ to $3.49 \mathrm{~mm}$, as shown in Fig. 9(c). In the case of the sliding test, $\Delta R_{S} / R$ shows a drastic increase to a peak value after beginning to slide, because of the static frictional force, as shown in Fig. 10. The peak value of $\Delta R_{S} / R$ increases with hardness and surface roughness [Figs. 10(a), and 10(c), respectively] and decreases with thickness [Fig. 10(b)] of the object. After the peak of $\Delta R_{S} / R$, there is a gradual downward trend, as shown in Figs. 10(a) and 10(b). On the other hand, $\Delta R_{S} / R$ shows periodic change for the objects with larger roughness $\left(R_{z}=2.14\right.$ and $\left.3.49 \mathrm{~mm}\right)$, as shown in the inset of Fig. 10(c).

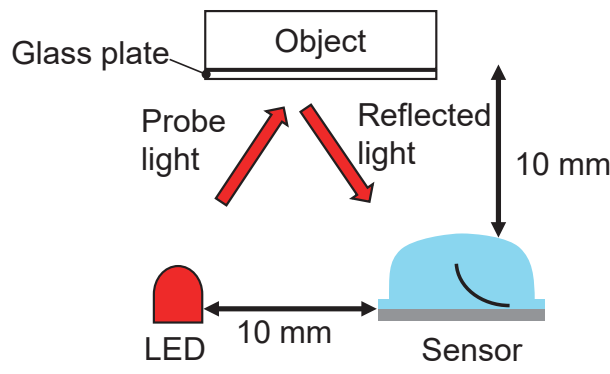

(a)

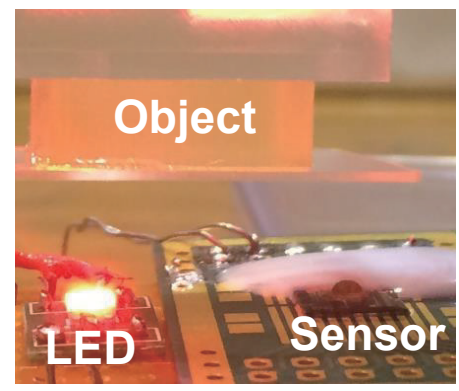

(b)

Fig. 8. (Color online) (a) Schematic illustration and (b) photograph of the measurement setup for the optical characterization of the object. 


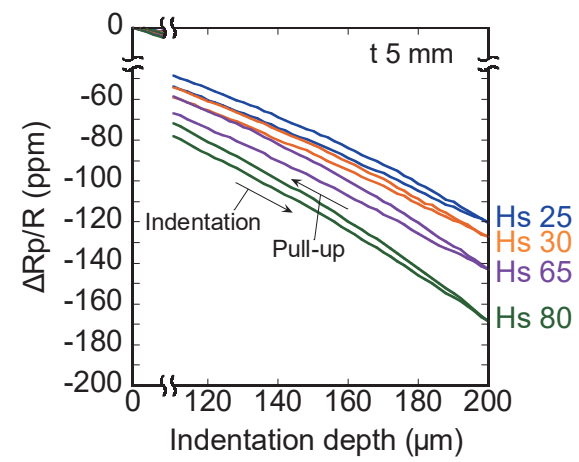

(a)

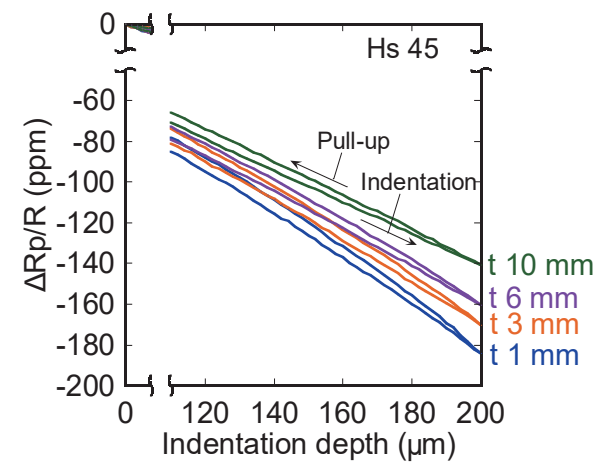

(b)

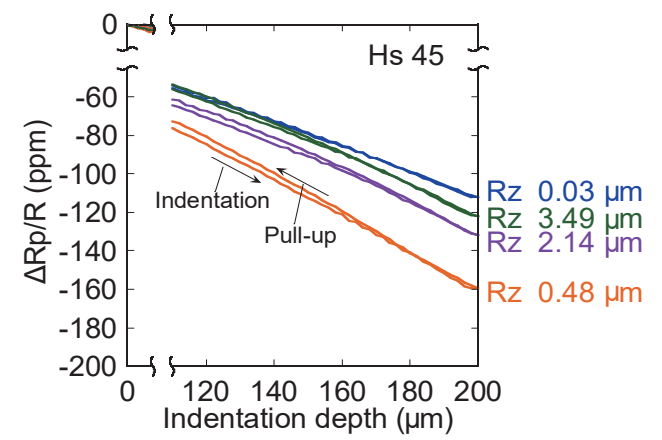

(c)

Fig. 9. (Color online) Changes in relative resistance of the sensor as a function of time when the objects are indented on the sensor and then pulled up in cases of various (a) hardness, $H s$, (b) thickness, $t$, and (c) surface roughness, $R_{z}$.

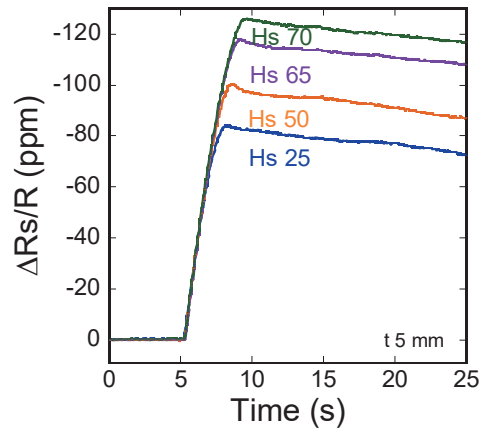

(a)

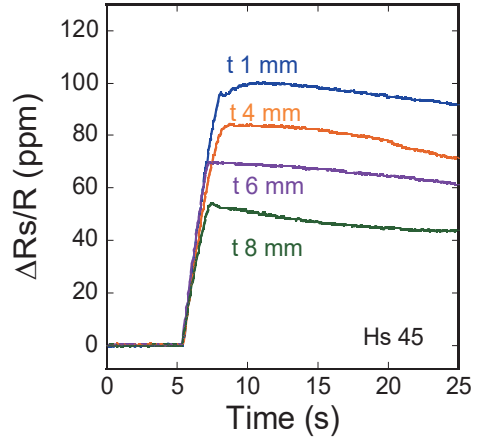

(b)

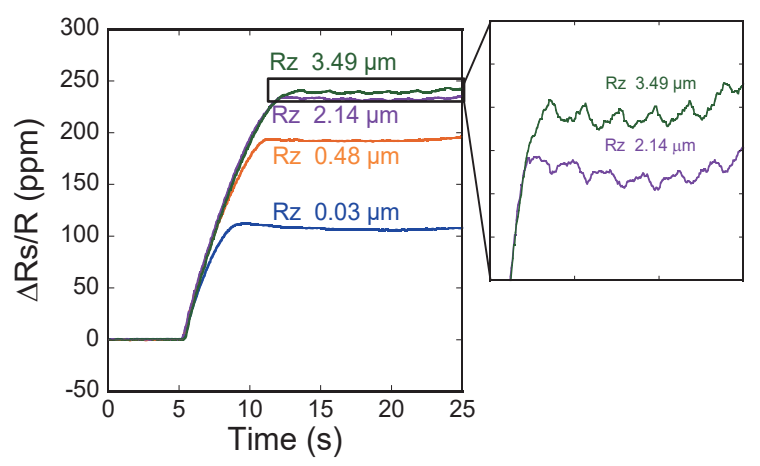

(c)

Fig. 10. (Color online) Changes in relative resistance of the sensor as a function of time when the sensor is slid on the objects with various (a) hardness, $H s$, (b) thickness, $t$, and (c) surface roughness, $R_{z}$. 
Resistance changes in Figs. 9 and 10 are schematically summarized in Fig. 11. We extracted four feature values from resistance changes for the evaluation of tactile texture. $\left(\Delta R_{p} / R\right)_{\max }$, $\left(\Delta R_{S} / R\right)_{\text {peak }}$, and $\left(\Delta R_{S} / R\right)^{\prime}{ }_{\text {max }}$ are the maximum decrease of $\Delta R_{p} / R$, the peak value of $\Delta R_{S} / R$, and the maximum gradient of $\Delta R_{S} / R$ as the sensor begins to slide, respectively. $\sigma_{\mathrm{s}}$ is the standard deviation of $\Delta R_{S} / R$ after its peak value. Scatter plots and coefficients of correlation between standardized values of these feature values $\left[\left(\Delta R_{p} / R\right)_{\max },\left(\Delta R_{S} / R\right)_{\text {peak }},\left(\Delta R_{S} / R\right)^{\prime}\right.$ max , and $\left.\sigma_{S}\right]$ and characteristics of the object (hardness, thickness, and surface roughness) obtained by regression analysis are shown in Fig. 12 and Table 1, respectively. Note that the coefficient of correlation

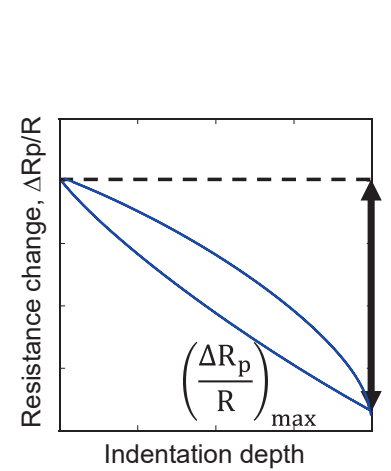

(a)

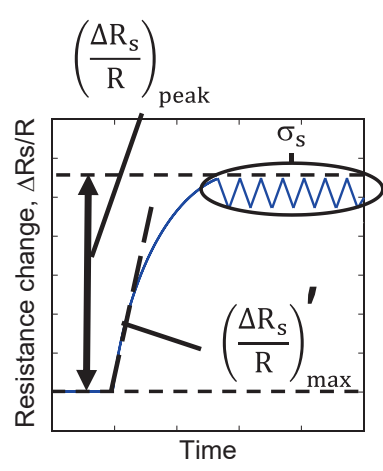

(b)

Fig. 11. (Color online) Four feature values for the evaluation of tactile texture extracted from resistance change in (a) indentation and pull-up test and (b) sliding test.

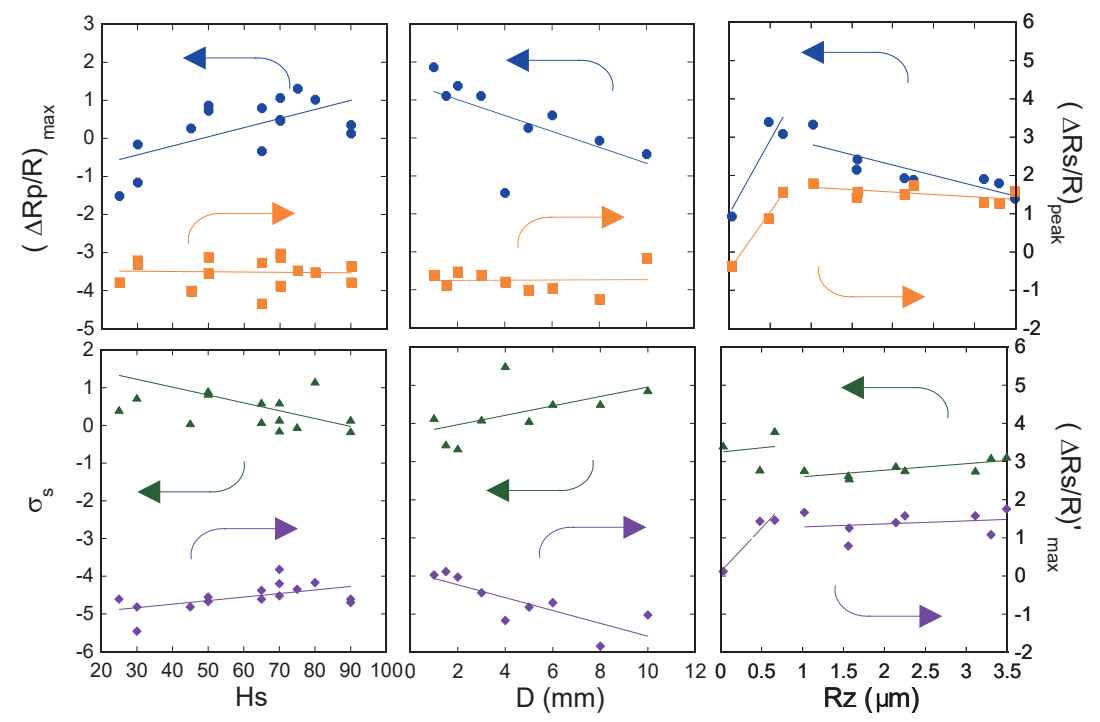

Fig. 12. (Color online) Scatter plots between four standardized feature values and characteristics of the object.

Table 1

Coefficients of correlation between feature values and characteristics of the object. Numbers shown in parentheses are $p$-value of each correlation.

\begin{tabular}{lrrrr}
\hline & $\left(\frac{\Delta R_{p}}{R}\right)_{\max }$ & $\left(\frac{\Delta R_{s}}{R}\right)_{\text {peak }}$ & \multicolumn{1}{c}{$\sigma_{s}$} & $\left(\frac{\Delta R_{S}}{R}\right)_{\max }^{\prime}$ \\
\hline Hardness & $0.625(0.013)$ & $-0.024(0.932)$ & $-0.490(0.064)$ & $0.540(0.038)$ \\
Thickness & $-0.629(0.070)$ & $0.008(0.984)$ & $0.563(0.114)$ & $-0.801(0.009)$ \\
$R_{z}<1 \mu \mathrm{m}$ & $0.922(0.253)$ & $0.997(0.049)$ & $0.140(0.911)$ & $0.966(0.167)$ \\
$R_{z}>1 \mu \mathrm{m}$ & $-0.842(0.009)$ & $-0.561(0.148)$ & $0.758(0.029)$ & $0.217(0.606)$ \\
\hline
\end{tabular}


for $R_{z}>1 \mathrm{~mm}$ was obtained separately from that for $R_{z}<1 \mathrm{~mm}$. There are obviously positive or negative correlations with $p$-value $<0.05$ between some feature values and characteristics of the object, such as $\left(\Delta R_{p} / R\right)_{\max }$ and hardness. $\left(\Delta R_{p} / R\right)_{\max }$ has a positive correlation $(0.625)$ and strong negative correlation $(-0.842)$ to hardness and $R_{z}>1 \mathrm{~mm}$, respectively. The former is because a harder sample applies stronger reaction force to the sensor and resistance reduction is increased with indentation. On the other hand, the latter is because the total reaction force received from a rougher sample with a smaller contact area is smaller. Furthermore, $\left(\Delta R_{S} / R\right)_{\text {peak }}$ shows strong positive correlation to $R_{z}<1 \mathrm{~mm}$, because the static friction coefficient depends on surface roughness. Also, there is a positive correlation (0.758) between $\sigma_{s}$ and $R_{z}>1 \mathrm{~mm}$. This is caused by the fluctuation of resistance due to roughness during sliding. $\left(\Delta R_{S} / R\right)^{\prime} \max$ negatively correlates $(-0.801)$ with the thickness of samples. It is considered that shear deformation of a thick sample is larger than that of a thin one, thus, shear force is absorbed and the time until the start of slip becomes long. As described above, four feature values well reflect the conditions when the sensor and the sample are in contact with each other. Therefore, we can quantitatively characterize the tactile texture of the object by analyzing these feature values extracted from resistance changes of the fabricated sensor.

\subsection{Characterization of optical features}

Figure 13 shows the impedance change of the sensor induced by three-color lights reflected from rubber samples of various colors relative to those without light irradiance. These samples have similar hardness $(H s \sim 70)$ and thickness $(5 \mathrm{~mm})$. For silicone rubber of a light color, the decrease in impedance is larger than that for other rubber samples. On the other hand, the decrease in impedance is comparatively small for the dark-colored natural and nitrile rubber samples. These results reflect the difference in the surface reflectivity on rubber because impedance decreases with the increase in light illuminance, as shown in Fig. 3. Furthermore, the decrease in impedance depends on the color of the irradiated light in the case of urethane rubber. When the irradiated light is blue or green, the decrease in impedance is smaller than when it is red. The hue of urethane rubber is estimated to be $38^{\circ}$ using the conventional image

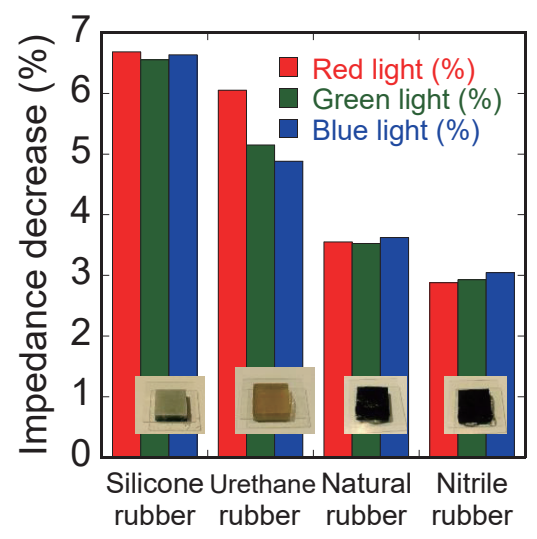

Fig. 13. (Color online) Relative impedance change of the sensor, induced by reflected light from rubber of various colors. 
sensor; thus, the impedance change upon irradiation with blue or green light is small, as shown in Fig. 4. It is suggested that the impedance change due to reflected light depends on the surface color of the material. Although the objects have similar tactile texture (hardness, thickness, and surface roughness), the decrease in impedance differs depending on the color of the rubber. However, it should be considered that the reflection light from the sample depends on surface roughness, hence we will study the effect of surface roughness on the optical feature in future work. Furthermore, spatial resolution of this measurement is limited by the spread of probe light and the distance between the LED and sensor. To improve spatial resolution, directivity of the probe light will be enhanced and the LED chip will be surface-mounted on the sensor in future work.

\section{Conclusions}

A MEMS sensor with force and light sensitivities was fabricated and applied to quantitative measurement of the surface texture of objects, including tactile and optical features. In the case of measurement with indentation and sliding of the sensor on the object, it was found that the resistance change of the strain gauge on the microcantilever embedded in the elastomer depends on the hardness, thickness, and surface roughness $\left(R_{z}\right)$, which constitute the tactile features of the object. The results of regression analysis suggested that feature values determined from the resistance change of the sensor have a high correlation with the above characteristics of the object. On the other hand, the impedance change with reflected light from the object depends on the color of the object. Therefore, it was demonstrated that multimodal texture can be characterized by the fabricated MEMS sensor. Although tactile and optical features are separately characterized in this work, our final goal is real-time simultaneous measurement of these features using this sensor installed in a robotic fingertip. We have succeeded in the simultaneous detection of force and color change in further work, ${ }^{(36)}$ and we believe that tactile and optical features can be characterized simultaneously in future work.

\section{Acknowledgments}

A part of this work was supported by the N.S Promotion Foundation for Science of Perception.

\section{References}

1 Y. Shimizu, T. Sadoyama, M. Kamijo, S. Hosoya, M. Hashimoto, T. Otani, K. Yokoi, Y. Horiba, M. Takatera, M. Honywood, and S. Inui: Int. J. Cloth. Sci. Tech. 16 (2004) 32.

2 C. Spence, M. E. R. Nicholls, N. Gillespie, and J. Driver: Percept. Psychophys. 60 (1998) 544.

3 R. Kawashima, J. Watanabe, T. Kato, A. Nakamura, K. Hatano, T. Schormann, K. Sato, H. Fukuda, K. Ito, and K. Zilles: Euro. J. Neurosci. 16 (2002) 137.

4 V. G. Chouvardas, A. N. Miliou, and M. K. Hatalis: Displays 29 (2008) 185.

5 K. Kim, M. Takatera, A. Zhu, and T. Otani: Autex Res. J. 15 (2015) 67.

6 R. S. Dahiya, G. Metta, M. Valle, and G. Sandini: IEEE Trans. Rob. 26 (2010) 1.

7 A.Takagi, Y. Yamamoto, M. Ohka, H. Yussof, and S. C. Abdullah: Proc. Comput. Sci. 76 (2015) 95.

8 N. Thanh-Vinh, N. Binh-Khiem, H. Takahashi, K. Matsumoto, and I. Shimoyama: Sens. Actuators, A 215 (2014) 167. 
9 A. Spanu, L. Pinna, F. Viola, L. Seminara, M. Valle, A. Bonfiglio, and P. Cosseddu: Org. Electron. 36 (2016) 57.

10 G. Liang, Y. Wang, D. Mei, K. Xi, and Z. Chen: J. Microelectromech. Syst. 24 (2015) 1510.

11 P. Goethals, H. Chaobal, D. Reynaerts, and D. Schaner: J. Clin. Monit. Comput. 28 (2014) 179.

12 A. M. Mazid and R. A. Russell: 2006 IEEE Conf. Robotics, Automation and Mechatronics (IEEE, 2006) 1.

13 D. Tsuchimi, T. Okuyama, and M. Tanaka: 13th Int. Conf. Biomedical Engineering, Eds. C. T. Lim and J. G. C. Hong (Springer-Verlag, Berlin, Heidelberg, 2009) 2219.

14 E.Asaga, K. Takemura, T. Maeno, A. Ban, and M. Toriumi: Sens. Actuators, A 203 (2013) 69.

15 T. Abe, T. Okatani, and K. Deguchi: IEEE Int. Conf. Pattern Recognition (IEEE, 2012) 3712.

16 I. Motoyoshi, S. Nishida, L. Sharan, and E. H. Adelson: Nature 447 (2007) 206.

17 H. Onishi, M. Sohgawa, H. Tachibana, Y. M. Huang, T. Kanashima, M. Okuyama, K. Yamashita, M. Noda, and H. Noma: IEEJ Trans. Sens. Micromach. 129 (2009) 411.

18 H. Tachibana, S. Kamanaru, T. Mima, M. Sohgawa, T. Kanashima, M. Okuyama, K. Yamashita, M. Noda, H. Noma, and M. Higuchi: IEEJ Trans. Sens. Micromach. 130 (2010) 223.

19 D. Hirashima, T. Uematsu, M. Sohgawa, W. Mito, T. Kanashima, M. Okuyama, and H. Noma: Jpn. J. Appl. Phys. 50 (2011) 06GM02.

20 M. Sohgawa, T. Uematsu, W. Mito, T. Kanashima, M. Okuyama, and H. Noma: Jpn. J. Appl. Phys. 50 (2011) 06GM08.

21 M. Sohgawa, D. Hirashima, Y. Moriguchi, T. Uematsu, W. Mito, T. Kanashima, M. Okuyama, and H. Noma: Sens. Actuators, A 186 (2012) 32.

22 H. Yokoyama, M. Sohgawa, T. Kanashima, T. Azuma, M. Okuyama, and H. Noma: Jpn. J. Appl. Phys. 52 (2013) 06GL08.

23 H. Yokoyama, T. Kanashima, M. Okuyama, T. Abe, H. Noma, T. Azuma, and M. Sohgawa: IEEJ Trans. Sens. Micromach. 134 (2014) 58

24 K. Watanabe, M. Sohgawa, T. Kanashima, M. Okuyama, and H. Noma: 2013 World Haptics Conf. (IEEE, 2013) 139.

25 M. Sohgawa, K. Watanabe, T. Kanashima, M. Okuyama, H. Noma, and T. Azuma: IEEJ Trans. Sens. Micromach. 133 (2013) 147.

26 K. Watanabe, M. Sohgawa, T. Kanashima, M. Okuyama, H. Noma, and T. Azuma: 17th Int. Conf. Solid-State Sensors, Actuators and Microsystems (IEEE, 2013) 1012.

27 M. Sohgawa, K. Watanabe, T. Kanashima, M. Okuyama, T. Abe, H. Noma, and T. Azuma: IEEE Sensors 2014 (IEEE, 2014) p. 1706.

28 M. Sohgawa, A. Nozawa, H. Yokoyama, T. Kanashima, M. Okuyama, T. Abe, H. Noma, and T. Azuma: IEEE Sensors 2014 (IEEE, 2014) 1749.

29 H. Yokoyama, T. Kanashima, M. Okuyama, T. Abe, H. Noma, T. Azuma, and M. Sohgawa: IEEJ Trans. Sens. Micromach. 134 (2014) 229.

30 M. Sohgawa: 2014 IEEE Int. Nanoelectronics Conf. (IEEE, 2014).

31 K. Takahashi, T. Abe, M. Okuyama, H. Noma, and M. Sohgawa: IEEE Sensors 2015 (IEEE, 2015) 699.

32 K. Takahashi, T. Abe, M. Okuyama, H. Noma, and M. Sohgawa: IEEE Sensors 2016 (IEEE, 2016) 655.

33 F. Sato, K. Takahashi, T. Abe, M. Okuyama, H. Noma, and M. Sohgawa: Sens. Mater. 29 (2017) 311.

34 T. K. Shih, C. F. Chen, J. R. Ho, and F. T. Chuang, Microelectron. Eng. 83 (2006) 2499.

35 S. M. Sze: Semiconductor Devices: Physics and Technology (John Wiley \& Sons, Inc., 2002) p. 287.

36 S. Sato, Y. Abe, T. Abe, H. Noma, and M. Sohgawa: 34th Sensor Symp. Sensors, Micromachines and Applied Systems (IEEJ, 2017) 31pm3-PS-22. 